\title{
Automated Classification of Congressional Legislation
}

\author{
Stephen Purpura \\ John F. Kennedy School of Government \\ Harvard University \\ +1-617-314-2027 \\ stephen_purpura@ksg07.harvard.edu
}

\author{
Dustin Hillard \\ Electrical Engineering \\ University of Washington \\ +1-206-789-1029 \\ hillard@ee.washington.edu
}

\begin{abstract}
For social science researchers, content analysis and classification of United States Congressional legislative activities has been time consuming and costly. The Library of Congress THOMAS system provides detailed information about bills and laws, but its classification system, the Legislative Indexing Vocabulary (LIV), is geared toward information retrieval instead of the pattern or historical trend recognition that social scientists value. The same event (a bill) may be coded as about $1,2,3$, or 12 subjects, with little indication of its primary emphasis. In addition, because the LIV system has not been applied to other activities, it cannot be used to compare (for example) legislative issue attention to executive, media, or public issue attention.
\end{abstract}

This paper presents the Congressional Bills Project's (www.congressionalbills.org) automated classification system. This system applies a topic spotting classification algorithm to the task of coding legislative activities into one of 226 subtopic areas. The algorithm uses a traditional bag-of-words document representation, an extensive set of human coded examples, and an exhaustive topic coding system developed for use by the Congressional Bills Project and the Policy Agendas Project (www.policyagendas.org). Experimental results demonstrate that the automated system is about as effective as human assessors, but with significant time and cost savings. The paper concludes by discussing challenges to moving the system into operational use.

\section{Categories and Subject Descriptors}

H.3.3 [Information Search and Retrieval]: Clustering, Information Filtering, Retrieval Models

\section{General Terms}

Algorithms, Performance, Experimentation

\section{Keywords}

U.S. Congress, legislative activities, text analysis, SVMs, support vector machines, institutions.

\section{INTRODUCTION}

The Congressional Bills Project received NSF funding in 2000 (SES 0080061) to assemble a dataset (www.congressionalbills.org) of all federal public bills introduced since 1947. The project contains 390,000 records that include details about the bill's substance, progress and its sponsor. Each bill is also assigned a single topic code drawn from the 226 subtopics of the Policy Agendas Project (www.policyagendas.org). The resulting database is of high quality and used by researchers, instructors, students and citizens to study relative policy attention across time and venues. Researchers on other project teams are classifying other government, media and public activities according to the same system, expanding the scope of comparison. A subset of published research, including articles and books, which consume the data may be found at:

http://www.policyagendas.org/publications/index.html.

At this time, a common classification scheme from the Policy Agendas Project makes possible comparisons of all Congressional bill activity with all Congressional hearings activity, Presidential State of the Union addresses, New York Times stories (sample), Solicitor General Briefs, and Gallup's Most Important Problem poll indices, among others for the period 1947-present.

To date, these classification efforts have depended on the efforts of trained human coders. However, the time and cost involved in expanding to new datasets and continually updating existing systems are substantial. A high quality, automated approach, especially one that allows lessons learned in one venue to be applied to another, would greatly speed the availability of the data to researchers.

The availability of high volume collections of high quality electronic documents describing government activity is a relatively new state of affairs for researchers. Not surprisingly, attempts at the automated sorting of this information at scale are few. Recent research from Laver [7] has examined automated classification of issue appeals in party platforms using a few techniques, most recently WordScores. In addition, Shulman and others [6][12] has examined regulatory comment email duplicate detection using Kullback-Leibler (KL) distance and clustering techniques. Although Shulman's work is closer to our approach, we will instead propose a general purpose method borrowed form research in newswire data topic spotting in computational linguistics.

Initially at least, legislative bills have similar document characteristics to newswire data. Topic spotting in legislative bills has similar goals to topic spotting in newswire data because both involve scanning a text segment for the predominance of a theme. Numerous techniques for topic classification have been well documented. In this work, support vector machines (SVMs) are chosen due to their strong performance on a wide variety of tasks. 
SVMs are a natural fit for topic classification because they deal well with sparse data and large dimensionality. Our only departure from standard topic classification research is that legislative text has different language patterns and characteristics from the typical news stories or broadcasts usually used in newswire topic spotting. Unlike news stories or broadcasts, legislative text uses a standard template and the language may be very similar for specific types of bills. However, one caveat is also immediately observable - not all legislation has few irrelevant features. Managing this problem will be discussed in future research. We propose the commonalities will overwhelm the difficulties and make the task of topic spotting in legislation quite successful.

The remainder of this paper documents our approach to building a prototype of a SVM system to sort the legislative text of the U.S. Congress using the Policy Agendas coding scheme and human coded samples. The approach was tested on roughly 108,000 of the 390,000 records in the Congressional Bills Project databases, as this was the largest sample available at the time of analysis. The approach to classifier design is developed in Section 2. The evaluation methodology is presented in Section 3. Experimental results are detailed in Section 4, and the main conclusions of this work are summarized in Section 5.

\section{ALGORITHM OVERVIEW}

Our goal is a software system that assists the Congressional Bills Project in classifying bills from the U.S. Congress according to the Policy Agendas coding scheme. Based on training examples (known as 'the truth') from expert coders, the system should scan each bill and determine, based on the examples of the truth, which of 226 subtopic codes best fits each bill. The section below describes an algorithm which accomplishes those objectives.

\subsection{Support Vector Machines}

SVMs were introduced in [14] and the technique attempts to find the best possible surface to separate positive and negative training samples. The best possible surface produces the greatest possible margin among the boundary points.

SVMs were developed for topic classification in [4]. Joachims motivates the use of SVMs using the characteristics of the topic classification problem: a high dimensional input space, few irrelevant features, sparse document representation, and that most text categorization problems are linearly separable. All of these factors are conducive to using SVMs because SVMs can train well under these conditions. That work performs feature selection with an information gain criterion and weights word features with a type of inverse document frequency. Various polynomial and RBF kernels are investigated, but most perform at a comparable level to (and sometimes worse than) the simple linear kernel. A software package for training and evaluating SVMs is available

Permission to make digital or hard copies of all or part of this work for personal or classroom use is granted without fee provided that copies are not made or distributed for profit or commercial advantage and that copies bear this notice and the full citation on the first page. To copy otherwise, or republish, to post on servers or to redistribute to lists, requires prior specific permission and/or a fee.

Conference'04, Month 1-2, 2004, City, State, Country.

Copyright 2004 ACM 1-58113-000-0/00/0004 ...\$5.00. and described by [5]. That package is used for these experiments.

\subsection{Word Feature Processing}

Text input to topic classification systems is usually preprocessed and then word features are given weights depending on importance measures. Most text classification work starts with word stemming to remove variable word endings and reduce words to a canonical form so that different word forms are all mapped to the same token (which is assumed to have essentially equal meaning for all forms). Word features usually consist of stemmed word counts, adjusted by some weighting. Inverse document frequency is commonly used, and has some justification [8]. More complex measures of word importance have shown to provide additional gains though. A weighted inverse document frequency is an extension of inverse document frequency to incorporate term frequency over texts, rather than just term presence [11].

Term selection can also help improve results and many past approaches have found information gain to be good criteria ([13] and [10].

\subsection{Hierarchical Approach}

Unlike our previous research in classifying political text, we will expose the powerful flexibility of our choice of SVMs by using a two-phase hierarchical approach to SVM training. This two phase approach mimics the method employed by human coders, who first classify a bill as falling under one of 20 major topic codes (see Table 1) and then further classify it as falling under one of 226 subtopics. For example, a bill proposing to reform the health care insurance system is assigned to fall under subtopic 301, where the 3 indicate health, and the 01 indicates health insurance reform.

\section{Table 1: Major Topic Codes}

\begin{tabular}{|l|}
\hline 1 = Macroeconomics \\
\hline 2 = Civil Rights, Minority Issues, and Civil Liberties \\
\hline 3 = Health \\
\hline 4 = Agriculture \\
\hline 5 = Labor, Employment, and Immigration \\
\hline 6 = Education \\
\hline 7 = Environment \\
\hline 8 = Energy \\
\hline $10=$ Transportation \\
\hline $12=$ Law, Crime, and Family Issues \\
\hline $13=$ Social Welfare \\
\hline 14 = Community Development and Housing Issues \\
\hline $15=$ Banking, Finance, and Domestic Commerce \\
\hline $16=$ Defense \\
\hline $17=$ Space, Science, Technology, and Communications \\
\hline $18=$ Foreign Trade \\
\hline
\end{tabular}




\begin{tabular}{|l|}
\hline $19=$ International Affairs and Foreign Aid \\
\hline $20=$ Government Operations \\
\hline $21=$ Public Lands and Water Management \\
\hline $99=$ Other \\
\hline
\end{tabular}

The advantages of the two phase approach were many, but two reasons stand out. First, training SVMs on 226 subtopic codes across large numbers of bills is computationally expensive. Using this hierarchical approach greatly reduces the computational expense of the sorting. The hierarchical approach can also be implemented on a common laptop computer with a complete sorting of the full data set in much less than a day of processing. Second, human coders are more likely to disagree on subtopic coding than they are on major topic coding. However, when human coders disagreed on major topic, the disagreements were frequently systemic.

The first pass of our system trains a set of SVMs to assign one of 20 major topics to each bill. The second pass of the system trains SVMs to assign subtopics within a major class. For example, we take all bills that were first assigned the major topic of health (3) and then train a collection of SVMs on the health subtopics (300398). This results in 21 sets of SVMs being trained - the major category SVM and 20 subtopic SVMs. This may be confusing, but, in the case of a bill about health, the major category SVM basically works with the health subtopic SVMs to differentiate across the 19 health subtopics.

The final step is subtopic placement. In this step, we assess the predictions from the hierarchical evaluation to make our best guess prediction for a bill. For each bill, we apply the subtopic SVM classifiers from each of the top 3 predicted major topic areas. This gives us the top 3 most likely subtopics of the top 3 most likely major categories.

An example would be helpful (see Table 2). For a bill which is clearly about health, we might predict a likelihood of $97 \%$ that it is about health. The second most likely major topic might be agriculture, with a $0.8 \%$ probability and the third most likely might be foreign affairs, with a $0.1 \%$ probability. Within health, the system predicts the top 3 subtopic codes as 342 (alcohol abuse and treatment), 343 (illegal drug use, treatment and education), and 344 (drug and alcohol or substance abuse treatment). The system then predicts three subtopic codes for each of the other two top major topics. Once the system has narrowed the choices down to the top 9 subtopics, it selects the subtopic which is most likely to match the bill, regardless of the major topic score. In this case, it selects subtopic code of 342 because it has the highest score of the top 9 subtopics.

Table 2: Sample evaluation table for subtopic placement

\begin{tabular}{|l|l|l|l|l|}
\hline Category & $\begin{array}{l}\text { Likelihood } \\
\text { of Major } \\
\text { Category }\end{array}$ & $\begin{array}{l}\text { Likelihood } \\
\text { of } \\
\text { Subtopic 1 }\end{array}$ & $\begin{array}{l}\text { Likelihood } \\
\text { of } \\
\text { Subtopic 2 }\end{array}$ & $\begin{array}{l}\text { Likelihood } \\
\text { of } \\
\text { Subtopic 3 }\end{array}$ \\
\hline Health & 0.97 & 0.74 & 0.20 & 0.05 \\
\hline Agriculture & 0.008 & 0.004 & 0.001 & 0.001 \\
\hline Foreign & 0.001 & 0.001 & 0.000 & 0.000 \\
\hline
\end{tabular}

\begin{tabular}{|l|l|l|l|l|}
\hline Affairs & & & & \\
\hline
\end{tabular}

\section{EVALUATION METHODOLOGY}

In this case, evaluation of success is straightforward because high quality information which describes "the ground truth" is available. This section describes the data sets used in our experiments and our methodology assessing performance against human labelers.

\subsection{Data Sets}

This research was conducted using the Congressional Bills Project's public data set, available from www.congressionalbillsproject.org. At the time (April 2004), 'only' 108,000 records were available for analysis. All statistics are generated from the 108,000 record set.

For the purposes of testing, the 108,000 records were divided into two groups and processed using a "train on $50 \%$, test on $50 \%$ " methodology. To select the groups, random sampling without replacement was applied across all of the bills. The experiment was repeated many times, and the statistics were comparable. We reported the last run.

\subsection{Evaluation Metrics}

We use metrics common in topic spotting and clustering analysis work in our evaluation of performance. The usefulness of our system was measured by its ability to predict the truth for every record. For analysis convenience, we also summarize consistency with the truth by major topic and subtopic classifications. Finally, we report Cohen's Kappa and AC1 to assess inter-coder agreement with the human team, as described in [3][12].

Cohen's Kappa statistic is a standard metric used to assess intercoder reliability between two sets of results. Usually, the technique is used to assess results between two human coders, but the computational linguistic field uses the metric as a standard mechanism to assess agreement between a human and machine coder.

Cohen's Kappa statistic is defined as:

$\kappa=\frac{p(A)-p(E)}{1-p(E)}$

In the equation, $\mathrm{p}(\mathrm{A})$ is the probability of the observed agreement between the two assessments:

$p(A)=\frac{1}{N} \sum_{n=1}^{N} I\left(\right.$ Human $_{n}==$ Computer $\left._{n}\right)$

Where $\mathrm{N}$ is the number of examples, and $\mathrm{I}()$ is an indicator function that is equal to one when the two annotations (human and computer) agree on a particular example. $\mathrm{P}(\mathrm{E})$ is the probability of the agreement expected by chance: 


$$
p(E)=\frac{1}{N^{2}} \sum_{c=1}^{C}\left(\text { HumanTotal }_{c} \times \text { ComputerTotal }_{c}\right)
$$

Where $\mathrm{N}$ is again the total number of examples and the argument of the sum is a multiplication of the marginal totals for each category. For example, for category 3 , health, the argument would be the total number of bills a human coder marked as category 3 , times the total number of bills the computer system marked as category 3. This multiplication is computed for each category, summed, and then normalized by $\mathrm{N}^{2}$.

For reasons of bias documented by [3], computational linguists also use another standard metric named the $\mathrm{AC} 1$ statistic to assess inter-coder reliability. The AC1 statistic corrects for the bias of Cohen's Kappa by calculating the agreement by chance in a different manner. It has similar form:

$$
A C 1=\frac{p(A)-p(E)}{1-p(E)}
$$

But the $\mathrm{p}(\mathrm{E})$ component is calculated differently:

$$
p(E)=\frac{1}{C-1} \sum_{c=1}^{C}\left(\pi_{c}\left(1-\pi_{c}\right)\right)
$$

Where $\mathrm{C}$ is the number of categories, and $\pi_{\mathrm{c}}$ is the approximate chance that a bill is classified as category $\mathrm{c}$.

$\pi_{c}=\frac{\left(\text { HumanTotal }_{c}+\text { ComputerTotal }_{c}\right) / 2}{N}$

In this paper, we report both Cohen's Kappa and AC1 because the two statistics provide consistency with topic spotting research and most other research in the field. For coding problems of this level of complexity, a Cohen's Kappa or AC1 statistic of 0.70 or higher is considered to be very good agreement between coders.

\section{EXPERIMENTAL RESULTS}

The Congressional Bills Project assessed the system by whether it reliably predicted the same major topic and subtopic about as well as a human. These results are reported in Tables 3 through 6, and they express that the system is about as accurate as a trained human coder at identifying the major topic of a bill, and sometimes as accurate at identifying the subtopic of a bill, with some exceptions. ${ }^{1}$

The results in Table 3 illustrate that the system automatically determines the correct major category for over $80 \%$ of the bills. The single worst category is Category 99, which makes sense because this is an 'Other' category that was only used for bills that could not reasonably be assigned to any other category. Performance on other categories varies, but is mostly above $80 \%$

\footnotetext{
${ }^{1}$ The latest version of the codebook is available at:
} http://www.policyagendas.org/codebooks/topicindex.html correct. The single best category was Category 18, 'Foreign Trade' at almost 90\%. Excluding the 'Other' category, the most difficult category was Category 19, 'International Affairs and Foreign Aid' at only $68 \%$ correct. 
Table 3: Number of Bills Predicted Correctly by Major Category, including totals.

\begin{tabular}{|l|r|r|r|}
\hline Category & Correct & Possible & Percent \\
\hline Macroeconomics (1) & 4148 & 5481 & 75.68 \\
\hline Civil Rights ... (2) & 1682 & 2397 & 70.17 \\
\hline Health (3) & 7246 & 8200 & 88.37 \\
\hline Agriculture (4) & 3137 & 3703 & 84.72 \\
\hline Labor ... (5) & 5232 & 7323 & 71.45 \\
\hline Education (6) & 3131 & 3613 & 86.66 \\
\hline Environment (7) & 4108 & 4871 & 84.34 \\
\hline Energy (8) & 4128 & 4660 & 88.58 \\
\hline Transportation (10) & 4518 & 5378 & 84.01 \\
\hline Law, Crime ... (12) & 5417 & 6491 & 83.45 \\
\hline Social Welfare (13) & 5249 & 6080 & 86.33 \\
\hline Community ... (14) & 1851 & 2447 & 75.64 \\
\hline Banking ... (15) & 5261 & 6876 & 76.51 \\
\hline Defense (16) & 6255 & 7440 & 84.07 \\
\hline Space, Science (17) & 1500 & 1845 & 81.30 \\
\hline Foreign Trade (18) & 4127 & 4647 & 88.81 \\
\hline International (19) & 1613 & 2372 & 68.00 \\
\hline Government Op (20) & 13416 & 15607 & 85.96 \\
\hline Public Lands ... (21) & 6830 & 7894 & 86.52 \\
\hline Other (99) & 145 & 943 & 15.38 \\
\hline Total & 88994 & 108268 & 82.20 \\
\hline
\end{tabular}

Table 4 presents the overall statistics for categorization at the subtopic category level. The number of possible bills is slightly lower (only by $0.1 \%$ ) because our two tiered approach only hypothesizes minor categories within the top three major categories for each bill. This provides for significant computational savings, while missing only a negligible number of bills. The overall percentage of correct bills is lower than for the major categories, at $71 \%$, but this task is significantly harder with over 200 possible categories instead of about 20 for the major category case.

Table 4: Number of Bills Predicted Correctly for Subtopic Categories (totals only).

\begin{tabular}{|l|r|r|r|}
\hline Subtopic & Correct & Possible & Percent \\
\hline Total & 76800 & 108143 & 71.02 \\
\hline
\end{tabular}

Table 5 and Table 6 present the 15 best and worst individual minor category results. The single best category is 1807 'Tariff and Import Restrictions, Import Regulation.' Many of the minor categories that had a large number of examples had better performance in the end, probably because the SVM was better able to learn the category characteristics when more examples were available. The 15 worst categories are primarily those categories with very few examples, and often were again those categories that were 'Other' categories within a major topic (those ending in 99).

Table 5: Number of Bills Predicted Correctly for Subtopic Categories (best 15 subtopic categories)

\begin{tabular}{|l|r|r|r|}
\hline \multicolumn{1}{|c|}{ Category } & \multicolumn{1}{c|}{ Correct } & Possible & Percent \\
\hline Category 1807 & 2754 & 2974 & 92.60 \\
\hline Category 2030 & 322 & 351 & 91.74 \\
\hline Category 2015 & 3071 & 3378 & 90.91 \\
\hline Category 1003 & 1022 & 1155 & 88.48 \\
\hline Category 1301 & 520 & 591 & 87.99 \\
\hline Category 2012 & 1257 & 1447 & 86.87 \\
\hline Category 501 & 470 & 542 & 86.72 \\
\hline Category 402 & 1379 & 1594 & 86.51 \\
\hline Category 1002 & 623 & 721 & 86.41 \\
\hline Category 341 & 258 & 299 & 86.29 \\
\hline Category 1707 & 538 & 624 & 86.22 \\
\hline Category 803 & 1532 & 1783 & 85.92 \\
\hline Category 707 & 176 & 205 & 85.85 \\
\hline Category 2003 & 806 & 942 & 85.56 \\
\hline Category 2102 & 854 & 1009 & 84.64 \\
\hline Category 601 & 1397 & 1653 & 84.51 \\
\hline
\end{tabular}

Table 6: Number of Bills Predicted Correctly for Subtopic Categories (worst 15 subtopic categories)

\begin{tabular}{|l|r|r|r|}
\hline Category & Correct & Possible & Percent \\
\hline Category 103 & 0 & 17 & 0.00 \\
\hline Category 1399 & 0 & 39 & 0.00 \\
\hline Category 1598 & 0 & 6 & 0.00 \\
\hline Category 1899 & 0 & 14 & 0.00 \\
\hline Category 209 & 0 & 17 & 0.00 \\
\hline Category 2199 & 0 & 6 & 0.00 \\
\hline Category 344 & 0 & 42 & 0.00 \\
\hline Category 698 & 0 & 15 & 0.00 \\
\hline Category 1999 & 1 & 23 & 4.35 \\
\hline Category 1614 & 2 & 41 & 4.88 \\
\hline Category 899 & 1 & 17 & 5.88 \\
\hline Category 9999 & 65 & 863 & 7.53 \\
\hline Category 1099 & 2 & 26 & 7.69 \\
\hline Category 599 & 3 & 29 & 10.34 \\
\hline Category 299 & 2 & 19 & 10.53 \\
\hline
\end{tabular}




\subsection{Systems-to-Human Inter-coder Agreement}

The second set of calculations assessed inter-coder reliability, as calculated using Cohen's Kappa and AC1. We use a single coder to express the performance of the entire Congressional Bills team and note that in future research we will integrate the system as a coder within the team for testing.

TABLE 7: Cohen's Kappa and AC1, human team versus the system

\begin{tabular}{|l|c|c|c|}
\hline & $\mathbf{p ( A )}$ & $\mathbf{p ( E )}$ & Statistic \\
\hline $\begin{array}{l}\kappa \quad \text { for all } \\
\text { major topics }\end{array}$ & 0.822 & 0.069 & 0.809 \\
\hline $\begin{array}{l}\kappa \quad \text { for all } \\
\text { subtopics }\end{array}$ & 0.710 & 0.013 & 0.706 \\
\hline $\begin{array}{l}\text { AC1 for all } \\
\text { major topics }\end{array}$ & 0.822 & 0.049 & 0.813 \\
\hline $\begin{array}{l}\text { AC1 for all } \\
\text { subtopics }\end{array}$ & 0.710 & 0.004 & 0.709 \\
\hline
\end{tabular}

\section{CONCLUSION AND NEXT STEPS}

Researchers are now classifying government, media and public activities according to a common system to expand the scope of comparison across government institutions. These researchers, including those involved with the Congressional Bills Project, have a need to perform coding and content analysis on large corpuses of text. The shift from paper documents to electronic documents should make their job easier, but without new tools and methods, progress will be slow and expensive.

This research focused on the process of sorting United States Congressional bills using an established classification system. Extensive work by the Congressional Bills team set the benchmark for measuring an automated system. This paper demonstrates that support vector machines are effective for efficiently classifying Congressional bills. On some types of bills, the system has difficulty compared to an expert coder. But, in the balance, the algorithm is quite compact and robust, considering its effectiveness and the complexity of coding legislative text into one of 226 subtopics.

The described algorithm also displays another trait which is highly desirable for the task - it is easily extensible with additional features. The SVM system is capable of considering out-of-band data to aid in reaching a conclusion in text classification. In concrete terms, the system can easily be told to consider THOMAS LIV classifications, sponsor committee membership, and other relevant information when predicting the subtopic of a bill. Any hint which systemically improves the ability of the system to increase accuracy in a category could be added. With the correct tools, extending the system to improve its accuracy would then become an exercise for any political science student interested in taking up the task.

But, in today's terms, the issue of integration of the algorithm with the Congressional Bills team is still pending. The next step is to provide the team with the ability to use the system in their daily work to predict a bill's major and subtopic codes. Although the system cannot be trusted to generate a $100 \%$ accurate answer, it can be made to generate meaningful information to help the team understand when it is making a systemic, likely true prediction versus a wild guess. The team is applying for National Science Foundation funding to pursue this opportunity.

\section{ACKNOWLEDGMENTS}

Thanks to Dr. John Wilkerson for providing assistance with the Congressional Bills' data. Also, thanks to Dr. Stuart Shulman for encouraging us to submit this document.

\section{REFERENCES}

[1] N. Cristianini, J. Shawe-Taylor, and H. Lodhi. 2001. Latent semantic kernels. In C. Brodley and A. Danyluk, editors, Proceedings of ICML-01, 18th International Conference on Machine Learning, pages 66-73. Morgan Kaufmann Publishers, San Francisco, US.

[2] S. Deerwester et al. 1990. Indexing by latent semantic analysis. Journal of the AmericanSociety of Information Science, 41(6):391-407.

[3] K. Gwet. Kappa Statistic is not Satisfactory for Assessing the Extent of Agreement Between Raters. In Series: Statistical Methods For Inter-Rater Reliability Assessment, No. 1, April, 2002.

[4] T. Joachims. 1998. Text categorization with support vector machines: Learning with many relevant features. In Proceedings of theEuropean Conference on Machine Learning.

[5] T. Joachims. 1999. Advances in kernel methods - support vector learning. Making large-Scale SVM Learning Practical.

[6] N. Kwon, S.W. Shulman, and E.H. Hovy. (Under review). "Collective text analysis for eRulemaking." Proceedings of the Sixth National Conference on Digital Government Research. San Diego, CA.

[7] M. Laver, K. Benoit, and J. Garry, 2003. Extracting policy positions from political texts using words as data. In American Political Science Review 97(2).

[8] K. Papineni. 2001. Why inverse document frequency? In NAACL Proceedings, pages 25-32.

[9] M. F. Porter. 1980. An algorithm for suffix stripping. Program, 16(3):130-137.

[10] F. Sebastiani. 2002. Machine learning in automated text categorization. ACM Computing Surveys, 34(1).

[11] T. Tokunaga and M. Iwayama, 1994. Text categorization based on weighted inverse document frequency. Technical Report 94 TR0001, Department of Computer Science, Tokyo Institute of Technology.

[12] H. Yang, J. Callan, and S. Shulman. (Under review) "Next steps in near-duplicate detection for eRulemaking." Proceedings of the Sixth National Conference on Digital Government Research. San Diego, CA.

[13] Y. Yang and X. Liu. 1999. A re-examination of text categorization methods. In Proceedings of SIGIR-99, November. 
[14] V. Vapnic. 1995. The Nature of Statistical Learning Theory. Springer, New York, NY. 